\title{
Top five reasons for dental complaints during the coronavirus pandemic revealed
}

At the one year anniversary of the UK's first lockdown, the Dental Defence Union (DDU) has analysed the top five reasons for patients to raise complaints about pandemic dental practice. The DDU supported dental practitioners with over 500 advisory and complaint cases between the start of lockdown in March to the end of 2020. In the majority of cases, members wanted advice on an aspect of patient care in order to pre-empt problems. Just $9 \%$ of cases were about complaints, and of these treatment availability was the most common reason for a patient to be dissatisfied.

The top five reasons for complaints were:

1. Treatment availability - delays caused by the closing of practices and the limitations on patient numbers after practices reopened.

2. Dissatisfaction with treatment - allegations of poor treatment outcomes and delayed diagnosis due to the pandemic.

3. Fees and refund requests - patients requesting a refund or compensation for treatment delays or incomplete treatment.
4. Communication issues - patients who felt they had not been properly informed about delays, cancellations and arrangements for urgent care during lockdown.

5. COVID-secure measures - issues such as infection control, PPE and the use of face masks

However, the DDU says it anticipates that complaint numbers are likely to increase because of delays in treatment caused by the pandemic. John Makin, head of the DDU said: 'Most patients have been understanding about the unavoidable compromises in dental provision during the pandemic but practices still had to respond to a significant number of complaints. Unfortunately, the possibility of complaints and claims is likely to increase as patients experience the detrimental consequences of treatment delays or become frustrated by the continued restrictions on routine dental practice. This risk can best be mitigated with pro-active and consistent communication which will help to manage patient expectations.'

The DDU examined the cases to identify the most significant themes and to help dental professionals understand where difficulties can arise. Findings included: $\rightarrow 92 \%$ of files concerned general dental practice. The remainder were specialist dental practice, community and hospital dentistry.

$\rightarrow$ Dentists featured in $72 \%$ of the files with dental therapists and hygienists and specialists making up $18 \%$ and 5\% respectively.

Makin continued: 'When responding to patient complaints due to the pandemic, it is important to highlight the circumstances prevailing at the time of treatment. We advise dental professionals to refer to the relevant guidance, regulations and standard operating procedures that impacted upon the care provided. Context is vital.'

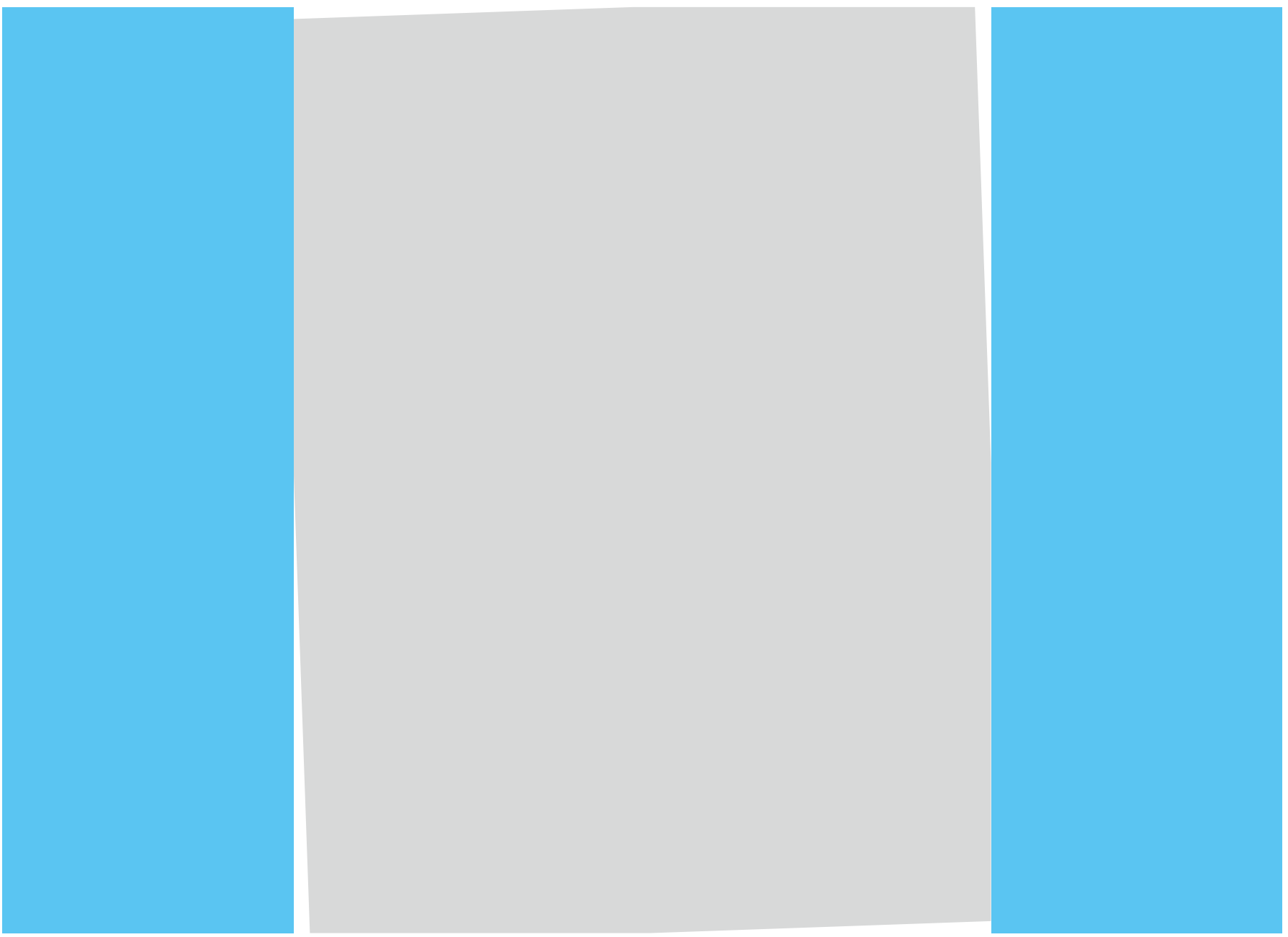

